\title{
Distributed hybrid P2P networking systems
}

\author{
Jungsoo Han
}

Published online: 20 June 2014

(C) Springer Science+Business Media New York 2014

Welcome to the special issue of Peer-to-Peer Networking and Applications. This issue contains a collection of the best papers out of various authors who have been submitted to this special issue. Most multimedia-based content service environments are embedded in a large-capacity server and adopt the client-server model. In such a model, excessive load on the server can slow down or break the network. This problem can be solved by applying a peer-to-peer (P2P) network in a distributed environment for smoother sharing and distribution of resources. Depending on the existence of an index server, the P2P models are categorized as hybrid peer-to-peer and pure peer-to-peer networks. A hybrid P2P network is one that has an index server containing information on the locations of resources at the center, and which uses the index server for search. A pure P2P network is one that directly executes the search process among hosts without a server between the hosts [9-11].

The main goal of this issue is to bring together researchers and practitioners in areas of media streaming, multicasting and multimedia delivery, P2P file sharing to share their visions, research achievements and solutions, to resolve the challenge issues and to establish worldwide cooperative researches and developments. This will give an opportunity to push further the discussion upon the potential distributed hybrid networking systems across many communities. Some of these research areas are listed below.

- Hybrid P2P networking system

- Knowledge based Applications and Management

- Multicasting and multimedia delivery

- P2P Overlay networks

- P2P Security issues

\section{J. Han $(\bowtie)$}

Division of Information \& Communication, Baekseok University, Cheonan City, Republic of Korea

e-mail: jungsoo.han.k@gmail.com
- P2P Wireless and mobile networks

- Architectures and protocols

- Convergence/co-existence of P2P and other networks

- Data and index structures

- Modeling, performance and robustness

- Semantic routing and search

- Systems Analysis, Design and Development

This special issue has become one of the hottest topics in distributed hybrid P2P networking systems. We believe that issue will have a high citation in the areas of Peer-to-Peer Networking and Applications.

The paper by Kim et al. [1] presents a quantum-dot-doped fiber amplifier (QDFA) that is used to obtain packet transmission times and packet arrival times during the QDFA synchronization process and which is a new framework for guaranteeing users with quality of service (QoS) from multimedia streaming services in hybrid P2P networks. In addition, the proposed QDFA utilizes IEEE 1588 technology, which is a packet network synchronization technique to accurately measure packet transmission time, and it ensures users' QoS based on this information. The paper by Joh et al. [2] presents a cross-layer system design for optimizing smart-device communication properties by utilizing the characteristics of highspeed data transmission rates for Wi-Fi P2P networks and the low power-consumption communication of Bluetooth. They also verified that the proposed technique outperforms existing Bluetooth and Wi-Fi P2P networks by comparing throughput as well as transmission delay through OPNET simulations, which leads to faster user connections and provides better QoS to end users.

The paper by Park et al. [3] suggests methods and a system to align content with annotation data to be used for TCommerce through a P2P-TV system while evaluating system performance. Experimentation on five different types of content corruption generated relatively strong annotation results of $82.63 \%$. The paper by Kim et al. [4] analyzes security 
services for secure multiple communications in $\mathrm{P} 2 \mathrm{P}$ networks, and proposes a key exchange process that provides PIM-SM-based authentication and confidentiality to securely join multiple communication services in $\mathrm{P} 2 \mathrm{P}$ networks.

The paper by Moon et al. [5] proposes a reliable information retrieval system for P2P services based in the mobile cloud environment. The proposed system is applied to the keyword-based concept net applying an ontology method. Additionally, for effective information retrieval by peers, connectivity between keyword concepts is applied. The paper by Oh et al. [6] presents an estimation method for abnormal objects and reliability in a P2P network. The proposed method is very useful for detection of abnormal objects in a P2P network that occur through abnormal faults in software and data. While they manage the P2P network, the reliability of the system can be predicted.

The paper by Kim et al. [7] presents a messenger real IP trace method that extracts the user-connected IP address or extracts an installed IP address in the computer via an agent. This can prevent crimes that make tracing impossible through use of a virtual private network (VPN) or a proxy server to hide the criminals' identities. Using the method proposed in this paper can prove to be a valuable method for the prevention of crime. The paper by Yun et al. [8] presents a biometric signature delegation scheme. The proposed scheme consists of biometric key-generation, signature delegation, and signature generation and verification protocols. The authors also show how the scheme could be applied to distribute content in a hybrid P2P network.

This fine collection of papers was achieved by fruitful collaborations. We wish to thank all the authors for their contributions and the reviewers for assisting our editorial work. We do hope that the papers included in this issue will satisfy the audience of the Peer-to-Peer Networking and Applications Journal and readers will find them interesting. Furthermore, we would like to thank Professor Sherman Shen, editor-in-chief of the international journals Peer-to-Peer Networking and Applications, for his valuable remarks and his undeterred help throughout the publication process of special issue.

\section{References}

1. Kim S (2014) QoS-aware data forwarding architecture for multimedia streaming services in hybrid peer-to-peer networks. Peer-to-Peer Netw Appl. doi:10.1007/s12083-014-0256-4
2. Joh H, Ryoo I (2014) A hybrid wi-fi P2P with bluetooth low energy for optimizing smart device's communication property. Peer-to-Peer Netw Appl. doi:10.1007/s12083-014-0276-0

3. Park S, Chung K (2014) Annotation alignment method on P2P-TV system. Peer-to-Peer Netw Appl. doi:10.1007/s12083-014-0264-4

4. Kim S, Kim B-H (2014) Key exchange process of PIM-SM-based for multiple group communication in P2P. Peer-to-Peer Netw Appl. doi: 10.1007/s12083-014-0274-2

5. Moon S, Yoon C-P (2014) Information retrieval system using the keyword concept net of the P2P service-based in the mobile cloud environment. Peer-to-Peer Netw Appl. doi:10.1007/s12083-0140265-3

6. Kim K, Hong M, Chung K, Oh S (2014) Estimating unreliable objects and system reliability in P2P networks. Peer-to-Peer Netw Appl. doi:10.1007/s12083-014-0257-3

7. Kim Y, Sun JH (2014) Extracting real IP address used to connect to P2P messenger using ARIT agent. Peer-to-Peer Netw Appl. doi:10. 1007/s12083-014-0275-1

8. Yun S, Lim H, Chung K (2014) The biometric signature delegation scheme to balance the load of digital signing in hybrid P2P networks. Peer-to-Peer Netwo Appl. doi:10.1007/s12083-014-0278-y

9. Song CW, Lee D, Chung KY, Rim KW, Lee JH (2013) Interactive middleware architecture for lifelog based context awareness. Multimed Tools Appl. doi:10.1007/s11042-013-1362-7

10. Boutaba R, Chung K, Gen M (2014) Recent trends in interactive multimedia computing for industry. Cluster Comput. doi:10.1007/ s10586-014-0349-0

11. Kim JH, Ryu JK (2013) Recent trends on high-performance computing and security. Cluster Comput 16(2):207-208. doi:10.1007/ s10586-013-0271-x

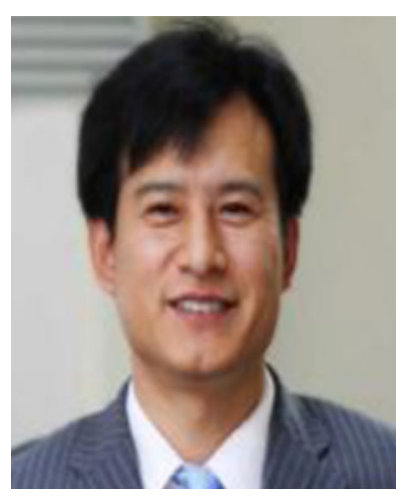

Jung-Soo Han received a BS, an $\mathrm{MS}$, and a PhD in Computer Engineering from Kyung Hee University, Republic of Korea. Since 2001, he has been a Professor in the Division of Information \& Communication, Baekseok University, Cheonan City, Chungnam, Republic of Korea. In 2014, he researched Convergence IT and Creative Education Methodology at California State University Fullerton as an Exchange Professor. His research topics include Data Mining, Contents Planning, 3D Modeling and CBD, Telemedicine, Knowledge-based Decision Support Systems, Intelligent Systems, Convergence, HCI, and Recommendation Systems. He has edited books on computer science and convergence technology. He serves as Executive Editing Director of the International Conference on Convergence Content (ICCC), as General Co-Chair of the International Conference on Digital Policy \& Management (ICPDM), as General Co-Chair for steering committees of the International Conference on Convergence Technology (ICCT), as Workshop Chair of the International Conference on Information Science and Application 2013, as Workshop Chair of the 2th International Conference on IT Convergence and Security 2012, as Vice President of the Korea Contents Association, as Vice President and a member of the Editorial Committee of the Society of Digital Policy \& Management, and as Vice President of the Editorial Committee of the Korea Contents Association. 